\title{
ESTUDO EXPERIMENTAL DE GRUPO ÚNICO COM AVALIAÇÃO PRÉ E PÓS: ATRAVÉS DA PRÁTICA DO ALONGAMENTO NA EXTENSÃO UNIVERSITÁRIA - FEF/UNICAMP
}

Gláucia Regina Falsarella

Marcy Garcia Ramos

Mauro Alexandre Páscoa

Fabrício Boscolo Del Vecchio

\section{Resumo}

Este trabalho abordou a atuação do alongamento como recurso modificador da aptidão física da flexibilidade e suas repercussões sobre a qualidade de vida após um período de 16 semanas. A população inicial envolvida no estudo foi constituída de 40 indivíduos e a amostra submetida às análises representaram 12 mulheres com faixa etária entre 22 e 42 anos de idade, envolvendo a participação da comunidade da Unicamp. Optou-se por desenvolver um estudo experimental, e como resultado observaram-se melhoras nas articulações de tronco e quadris, referentes aos aspectos da qualidade de vida, foram identificados escores expressivos para os domínios dor e aspectos sociais.

\section{Palavras-Chave}

Alongamento; Flexibilidade; Qualidade de vida.

EXPERIMENTAL STUDY OF A SINGLE GROUP WITH PRE AND POST ASSESSMENT: THROUGH THE PRACTICE OF STRETCHING THE UNIVERSITY EXTENSION FEF/UNICAMP

Gláucia Regina Falsarella

Marcy Garcia Ramos

Mauro Alexandre Páscoa

Fabrício Boscolo Del Vecchio

\begin{abstract}
This work approached the performance of Stretching as a modifier resource of the physical aptitude of flexibility and its repercussions on the quality of life after a period of 16 weeks. The population involved in the study was constituted of 40 individuals involving the participation of the community of the State University of Campinas - UNICAMP. The sample submitted to the analyses represented 12 women age between 22 and 42 years old. It was chosen to develop an experimental study, and as resulted it was observed improvements in the trunk joints and hips as well, referring to the aspects of the quality of life. It was also identified expressive scores for the domain of pain and social aspects.
\end{abstract}

\section{Key-Words}

Stretching; Flexibility; Quality of life 


\section{INTRODUÇÃO}

Considerada como elemento essencial para a funcionalidade do aparelho locomotor humano, a flexibilidade compreende um dos componentes da aptidão física relacionada à saúde e também é descrita por Alter (1999), como responsável pela execução de movimentos de amplitude angular máxima pelas articulações sem risco de provocar lesão.

A prática do alongamento como recurso modificador da flexibilidade, atua também na assistência à saúde em nível primário contra as algias, especialmente a relacionada ao segmento da coluna vertebral, e também como ação secundária esta prática corporal contribui à redução ou alívio da freqüência e intensidade da dor de inúmeros agravos osteomusculares, prevenção contra determinadas patologias do sistema locomotor, propiciando maior resistência às lesões, menor propensão quanto à incidência de dores musculares, principalmente na região dorsal e lombar, à medida que são minimizadas as ocorrências de problemas posturais (ALTER, 1999; WARBURTON et al., 2006).

As principais técnicas de alongamento para o desenvolvimento da flexibilidade incluem o alongamento Estático Ativo, Estático Passivo e (FNP) Facilitação Neuromuscular Proprioceptiva (ALTER, 1999).

Para o autor citado, o alongamento estático ativo se caracteriza pelo uso voluntário dos músculos de um indivíduo sem ajudas externas, sendo incapaz de exceder os limites fisiológicos. Já o método Estático Passivo constitui-se do alongamento que não necessita da contribuição ou contração ativa do indivíduo submetido à ação, mas utiliza-se de forças externas, através do auxílio de um companheiro ou de um equipamento.

O método denominado Facilitação Neuromuscular Proprioceptiva (FNP), caracteriza-se como uma técnica que incorpora diversas seqüências, em que estão envolvidos o relaxamento e contração dos músculos atuantes no processo do alongamento.

Nesta perspectiva, Achour Júnior (1995) destaca que a escassez de flexibilidade principalmente na região de tronco e quadril são apontadas como fator de risco para o desencadeamento de dores lombares. Cerca de $80 \%$ das lombalgias são causadas pela combinação de níveis de flexibilidade articular reduzidos, musculatura abdominal flácida e problemas posturais.

A efetividade de métodos preventivos, curativos e de reabilitação para as algias posturais encontra-se aqueles que consideram os exercícios de alongamento, como recurso terapêutico modificador da saúde ao 
demonstrarem resultados significativos na redução da freqüência e intensidade da dor (MARTINS, PINTO e SILVA, 2005).

Neste contexto, o objetivo deste estudo foi analisar a eficácia de um programa de alongamento sobre a amplitude articular e avaliar os indicadores de qualidade de vida da população envolvida nas intervenções.

\section{MATERIAL E MÉTODO}

A população inicial envolvida no estudo foi constituída de 40 indivíduos e a amostra submetida às análises representaram um total de 12 mulheres participantes das atividades de extensão da Faculdade de Educação Física da Unicamp, que treinavam três vezes por semana durante 1 hora. O universo do trabalho caracterizou-se por um grupo experimental com a prática do alongamento, com enfoque sobre os Métodos: Estático Ativo, Estático Passivo e Facilitação Neuromuscular Proprioceptiva (FNP).

Esse grupo foi analisado por um protocolo de avaliação em dois períodos distintos, pré e pós-testes, para obtenção do perfil dos participantes, dimensionar o grau da flexibilidade e os domínios da qualidade de vida.

Para analisar o grau de flexibilidade dos indivíduos, utilizamos o Flexímetro Carci $^{\circledR}$, instrumento que determina em graus, o nível de amplitude de movimento articular. A avaliação compreendeu os seguintes movimentos: flexão de ombros, tronco, quadris e joelhos.

Anexo aos testes de flexibilidade, foi aplicado o questionário SF-36 com questões relativas aos domínios integrantes da qualidade de vida da população participante da pesquisa.

Os dados obtidos nas avaliações da flexibilidade foram trabalhados segundo análise estatística disponível no Paired Student's T-Test: Results (2007).

A pesquisa e o termo de consentimento livre e esclarecido foram aprovados pelo Comitê de Ética da FCM-UNICAMP, sob o n 348/2006. 


\section{RESULTADOS}

$\mathrm{Na}$ tabela a seguir, observamos à mediana dos resultados da flexibilidade obtida no início e término do estudo, considerando a significância $\mathrm{p}<0,05$ para os movimentos de flexão das articulações dos ombros, tronco, quadris e joelhos.

Tabela 1- Mediana dos resultados da flexibilidade e ganhos da amplitude em porcentagem.

\begin{tabular}{lccc}
\hline \multicolumn{1}{c}{ Flexão } & Inicial & Final & Ganhos \% \\
Ombro Direito & $160( \pm 11,7)$ & $169( \pm 11,9)$ & 5.62 \\
\hline Ombro Esquerdo & $161( \pm 9,77)$ & $162( \pm 10,5)$ & 0.62 \\
Tronco & $91( \pm 12,2)$ & $97( \pm 11,7)$ & 6.59 \\
\hline Quadril Direito & $108( \pm 4,79)$ & $116( \pm 4,94)$ & 7.40 \\
Quadril Esquerdo & $112( \pm 5,84)$ & $116( \pm 4,01)$ & 3.57 \\
\hline Joelho Direito & $117( \pm 4,87)$ & $120( \pm 5,20)$ & 2.56 \\
Joelho Esquerdo & $118( \pm 5,96)$ & $120( \pm 5,96)$ & 1.69 \\
\hline
\end{tabular}

Analisaremos através do Quadro 1, escores padronizados dos participantes da pesquisa, os resultados obtidos para os vários domínios que compõem a qualidade de vida, no inicio e ao término do estudo, considerando seu nível de significância.

Quadro 1 - escores iniciais e finais dos participantes do estudo para os Domínios do SF - 36.

\begin{tabular}{|cccccc}
\hline & \multicolumn{2}{c}{ Escore Inicial } & Escore Final & \\
Domínio & Menor & Maior & Menor & Maior & Valor de p \\
Capacidade Física & 65 & 100 & 75 & 100 & 0,064 \\
Função Física & 0 & 100 & 25 & 100 & 0,041 \\
Dor Corporal & 41 & 84 & 61 & 100 & 0,007 \\
Saúde Geral & 47 & 95 & 57 & 97 & 0,208 \\
Vitalidade & 25 & 85 & 35 & 85 & 0,044 \\
Social & 38 & 100 & 50 & 100 & 0,006 \\
\hline
\end{tabular}

\section{DISCUSSÃO}

Os resultados obtidos no inicio e no final das intervenções mostram um aumento da mobilidade articular para o movimento de flexão dos ombros, tronco, quadris e joelhos. E neste sentido destaca-se a pesquisa de Teodoro (2004); Falsarella e Ramos (2007), que investigaram as alterações obtidas na flexibilidade 
após um programa de exercícios de alongamento e foram identificados adaptações com resultados positivos para a mobilidade das articulações em estudo.

Os resultados da presente pesquisa vão de encontro com os trabalhos científicos envolvendo a temática sobre alongamento, ao demonstrarem respostas favoráveis sobre a aptidão física da flexibilidade. E neste sentido Alter (1999), expressa a efetividade dos exercícios de alongamento como elemento atuante na flexibilidade, ao propiciar modificações na amplitude de movimento, devido a extensibilidade de músculos, ligamentos e tendões, que possibilita a obtenção ou manutenção de bons níveis deste componente da aptidão física.

Através da estatística descritiva dos escores do questionário SF-36, identificamos resultados positivos e expressivos para os domínios dor e aspectos sociais que compõem os indicadores de qualidade de vida.

Quanto ao domínio dor, os dados expressam uma alteração significativa para este elemento da qualidade de vida, após a aplicação dos exercícios. Nosso estudo confirma os resultados atingidos por Moraes (2003) em sua pesquisa ao demonstrar a efetividade das atividades executadas no programa educativo de exercícios físicos, em que se destaca o alongamento muscular sobre a qualidade de vida.

Diante deste quadro, Faria Junior, Barros (2004) destacam em seus estudos que as afecções que atingem os músculos e os seus ligamentos associados, outros tecidos conectivos e os ossos e cartilagens, podem ser prevenidas ou minimizadas através de um programa de exercícios de alongamento, ao estabelecer relações favoráveis entre o aumento da flexibilidade e o alívio de dores musculares e articulares decorrentes do processo de encurtamento músculo-tendíneos.

Após a aplicação de nossa pesquisa, verificamos a evolução satisfatória dos domínios Aspectos Sociais, e podemos afirmar que os benefícios do alongamento como experiência social caracteriza-se por propósitos socializadores, tendo o objetivo de ampliar, incentivar, ajudar e a perpetuar as relações sociais, ao repercutir sobre o convívio social, adaptação física-afetiva para obtenção e manutenção de um estilo de vida saudável (D’Ávila, 1999).

\section{CONSIDERAÇÕES FINAIS}

Este estudo evidenciou que o programa de exercícios de alongamento foi capaz de promover modificações na capacidade física da flexibilidade com destaque para as articulações de tronco de quadril 
e adaptações expressivas nos domínios dor e aspectos sociais que compõe os indicadores de qualidade de vida, após um período de 16 semanas.

Estes dados corroboram com a literatura sobre as repercussões positivas promovidas pela prática regular do alongamento, especialmente na esfera da saúde ao estabelecer uma associação direta desta prática corporal sobre a aptidão física para a promoção da saúde.

\section{REFERÊNCIAS}

ACHOUR JUNIOR, A. Exercícios de alongamento: anatomia e fisiologia. São Paulo: Manole, 1995.

ALTER, M. J. Ciência da flexibilidade. 2 ed. Porto Alegre: Artmed, 1999.

D’ÁVILA, F. Ginástica, dança e desporto para a terceira idade. Brasília: SESI-DN: INDESP, 1999.

FALSARELLA, G. R.; RAMOS, M. G. Alongamento uma prática saudável: estudo de sua influencia sobre a flexibilidade. FIEP Buletin, v.77, 2007.

FARIAS JÚNIOR, J. C.; BARROS, M. V. G. Flexibilidade e aptidão física relacionada à saúde. Corporis, Recife, v. 1, n. 1, 1998.

MARTINS, R. F.; PINTO E SILVA, J. L. An exercise method for the treatment of lumbar and posterior pelvic pain in pregnancy. Revista Brasileira Ginecologia e Obstetrícia, Rio de Janeiro, v. 27, n. 5, 2005.

MORAES, A. A. Avaliação da eficácia de um programa de reabilitação como modificador nos indicadores de dor e qualidade de vida em pacientes com lombalgias crônica inespecífica. 2003. Tese (Doutorado)- Faculdade de Educação Física, Universidade Estadual de Campinas, Campinas, 2003.

PAIRED Students t - test: results. Disponivel em: http://translate.google.com/translate?hl=pt$\mathrm{BR} \& \mathrm{sl}=\mathrm{en} \& \mathrm{u}=\mathrm{http}: / / \mathrm{www}$. physics.csbsju.edu/stats/Paired_t test_NROW_form.html\&sa $=\mathrm{X} \&$ oi $=$ translate\&resnum $=4 \& \mathrm{ct}=$ result\&prev $=/ \mathrm{search} \% 3 \mathrm{Fq} \% 3 \mathrm{Dtest} \% 2 \mathrm{Bt} \% 2$ 6hl\%3Dpt-BR\%26sa\%3DG, Acesso em: 2007.

THEODORO, P. F. R. Avaliação de um programa de treinamento da flexibilidade utilizado para compensação de esforços. 2004. Dissertação (Mestrado)- Faculdade de Educação Física- Universidade estadual de Campinas, Campinas, 2004.

WARBURTON, D. E. R.; NICOL, C. W.; BREDIN, S. S. D. Prescribing exercise as preventive therapy. Medicine and Science in Sport and Exercise, v. n. p. Canadá, 2006. 


\section{GLÁUCIA REGINA FALSARELLA}

Unicamp

\section{MARCY GARCIA RAMOS}

Unicamp

\section{MAURO ALEXANDRE PÁSCOA \\ UNICAMP}

\section{FABRÍCIO BOSCOLO DEL VECCHIO UNICAMP}

\section{Referência do artigo:}

\section{ABNT}

FALSARELLA, G. R. et. al. Estudo experimental de grupo único com avaliação pré e pós: através da prática do alongamento na extensão universitária. Conexões, v. 6, n. 1, p. 79-85.

\section{APA}

Falsarella, G. R., Ramos, M. G., Páscoa, M. A., \& Vecchio F. B. (2008). Estudo experimental de grupo único com avaliação pré e pós: através da prática do alongamento na extensão universitária. Conexões, 6(1), 79-85.

\section{VANCOUVER}

Falsarella GR, Ramos MG, Páscoa MA, Vecchio FB. Estudo experimental de grupo único com avaliação pré e pós: através da prática do alongamento na extensão universitária. Conexões, 2008, 6(1): 79-85. 